\title{
Tailoring Fluorescent Dyes To Optimize a Hybrid RGD-Tracer
}

\author{
Anton Bunschoten, ${ }^{\dagger}$ Danny M. van Willigen, ${ }^{\dagger}$ Tessa Buckle, ${ }^{\dagger}$ Nynke S. van den Berg, ${ }^{\dagger, \dagger}$ Mick M. Welling, ${ }^{\dagger}$ \\ Silvia J. Spa, Hans-Jürgen Wester, ${ }^{\dagger}$ and Fijs W. B. van Leeuwen ${ }^{\dagger}, \dagger,+$ \\ ${ }^{\dagger}$ Interventional Molecular Imaging Laboratory, Department of Radiology, Leiden University Medical Center, Albinusdreef 2, PO \\ BOX 9600, 2300 RC, Leiden, The Netherlands \\ "Departments of Urology and Head and Neck Surgery \& Oncology, The Netherlands Cancer Institute - Antoni van Leeuwenhoek \\ Hospital, Plesmanlaan 121, 1066 CX Amsterdam, The Netherlands \\ ${ }^{\S}$ Pharmaceutical Radiochemistry, Faculties of Chemistry and Medicine, Technische Universität München, 85748 Garching, Germany
}

\section{Supporting Information}

ABSTRACT: Quantitative assessment of affinity and kinetics is a critical component in the development of (receptor-targeted) radiotracers. For fluorescent tracers, such an assessment is currently not yet applied, while (small) changes in chemical composition of the fluorescent component might have substantial influence on the overall properties of a fluorescent tracer. Hybrid imaging labels that contain both a radiolabel and a fluorescent dye can be used to evaluate both the affinity (fluorescent label) and the in vivo distribution (radiolabel) of a targeted tracer. We present a hybrid label oriented and matrix-based scoring approach that enabled quantitative assessment of the influence of (overall) charge and lipophilicity of the fluorescent label on the (in vivo) characteristics of $\alpha_{\mathrm{v}} \beta_{3}$-integrin targeted tracers. Systematic chemical alterations in the fluorescent dye were shown to result in a clear difference in the in vivo distribution of the different hybrid tracers. The applied evaluation technique resulted in an optimized targeted tracer for $\alpha_{\mathrm{v}} \beta_{3}$-integrin, which combined the highest $\mathrm{T} / \mathrm{M}$ ratio with the lowest uptake in other organs. Obviously this selection concept would also be applicable during the development of other (receptor-targeted) imaging tracers.

\section{INTRODUCTION}

Clinical image-guided surgery trials suggest that a hybrid surgical guidance approach, which utilizes both the radioactive and fluorescent signatures of a single tracer (as demonstrated by, e.g., the sentinel node tracer indocyanine green- ${ }^{99 \mathrm{~m}} \mathrm{Tc}$ nanocolloid), ${ }^{1-3}$ holds great promise. ${ }^{4}$ For this concept to be suitable for the targeting of tumor-specific receptors, it needs to be expanded via the addition of in vivo vectors, such as peptide ligands, nanobodies, and/or antibody fragments. ${ }^{5}$

Peptides have proven to be fairly ideal imaging vectors since they can be produced at relatively low cost and they can be synthetically adapted. Moreover, they can be modified to target a range of biomarkers and imaging applications. ${ }^{6}$ Preferably, conjugation of an imaging label to the peptide-based vector should not negatively alter the compounds targeting capabilities and in vivo characteristics. Radioisotopes can be included in a relatively straightforward manner via either covalent introduction (e.g., ${ }^{18} \mathrm{~F}$; PET) or the incorporation of a chelate (e.g., DTPA or DOTA). The latter allows coordination of a radioisotope suitable for either PET (e.g., ${ }^{68} \mathrm{Ga}$ ) or SPECT (e.g., ${ }^{99 \mathrm{~m}} \mathrm{Tc}$ and ${ }^{111} \mathrm{In}$ ) imaging. For fluorescence imaging, incorporation of, or labeling with, a fluorescent dye is mandatory. Here, organic dyes are favored over, e.g., inorganic fluorescent quantum dots due to their smaller size $(<1 \mathrm{~nm})$, the absence of (possibly) toxic metals, and their ability to be tailored for conjugation to biomolecules. Yet, to date, "lack of toxicity" and "clinically approved" are often the predominant selection criteria for the conjugation of dyes (e.g., CW800 and ZW800) to in vivo vectors. The physical characteristics of the dye that relate to the signal intensity (e.g., quantum yield and molar absorption coefficient) and stability are seldom taken into account. Simultaneously, quantitative assessment of the effect that is exerted by a fluorescent label on the affinity and specificity of a (peptide-based) vector is commonly lacking as fluorescence-based assessment of the in vivo kinetics is hampered by tissue attenuation of both the excitation and emission light. $^{7-9}$ Such quantitative evaluation can be performed via radiolabeling.

We previously developed an $\alpha_{v} \beta_{3}$-integrin specific hybrid tracer that was functionalized with both ${ }^{111}$ In-DTPA and CyAL-5.5b. This tracer demonstrated a high tumor-to-muscle ratio (T/M-ratio; $9.02 \pm 3.81)$ and allowed image-guided resection of both the primary tumor and the distant metastases. $^{10}$ Unfortunately, in this tracer the fluorescent component largely dictated the in vivo characteristics, ${ }^{10}$ an effect that was also reported for other in vivo vectors. ${ }^{7,8,11-15}$ Further studies in this area are therefore warranted.

Received: February 18, 2016

Revised: April 10, 2016

Published: April 13, 2016 

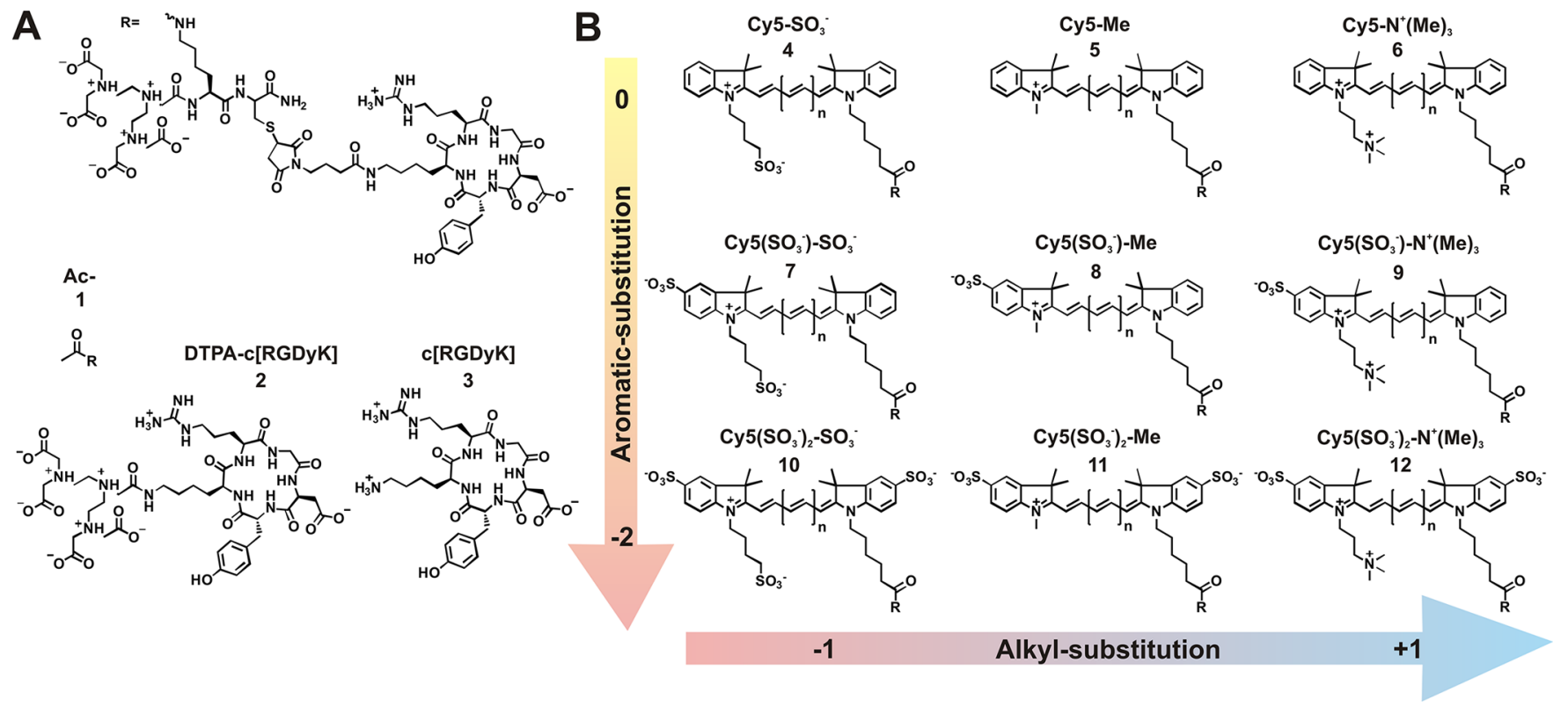

Figure 1. Structures of the $\alpha_{\mathrm{v}} \beta_{3}$-integrin targeted tracers. (A) Structures of the standardized hybrid tracer (top structure) and the three reference compounds used (1-3). (B) Incorporation of systematically modified Cy5-dyes $(n=1)$ into the hybrid label, resulting in tracers 4-12.

Integrins are transmembrane receptors that play an important role in cell-cell and cell-extracellular matrix interactions. ${ }^{16,17}$ Especially $\alpha_{\mathrm{v}} \beta_{3}$-integrin is involved in the repair of damaged vasculature and the formation of new blood vessels. ${ }^{17}$ Overexpression of $\alpha_{v} \beta_{3}$-integrin has been linked to several angiogenesis-related diseases, such as rheumatoid arthritis, osteoporosis, and cancer. ${ }^{18}$ In cancer, $\alpha_{\mathrm{v}} \beta_{3}$-integrin is linked to tumor invasiveness and the formation of metastases. ${ }^{19}$ $\alpha_{\mathrm{v}} \beta_{3}$-integrin has therefore been used as a biomarker for the development of optical ${ }^{20,21}$ and nuclear imaging tracers, of which some have been evaluated in clinical trials (e.g., ${ }^{99 \mathrm{~m}} \mathrm{Tc}$ NC100692, ${ }^{18} \mathrm{~F}$-Galacto-RGD, ${ }^{22}{ }^{18} \mathrm{~F}$-fluciclatide, ${ }^{23,24}$ and ${ }^{18} \mathrm{~F}$ alfatide). ${ }^{25}$ Cyclic RGD peptides (e.g., c $[$ RGDyK]) drive the receptor affinity in these structures. ${ }^{10,26,27}$ These peptides tolerate broad structural modifications of the lysine side chain while maintaining affinity for the target receptor. ${ }^{28}$

To quantitatively assess the influence of the fluorescent dye on the characteristics of a targeted tracer, we synthesized a range of hybrid tracers wherein the chemical design of the fluorescent label is the only variable. The synthesized hybrid $\mathrm{c}[\mathrm{RGDyK}]$-peptides were used for in vitro and in vivo targeting, and the in vivo characteristics of these tracers were evaluated via a matrix based scoring system.

\section{RESULTS AND DISCUSSION}

A matrix-based scoring system for $\alpha_{\mathrm{v}} \beta_{3}$-integrin targeted hybrid tracers was developed (Figure 1; Table 1). In this matrix the chemical design of the fluorescent dye was varied systematically. Of the available cyanine dyes (Cy3, Cy5, and Cy7), Cy5 $(n=1$; Figure 1$)$ was selected for this initial evaluation based on its recent successful use in an image-guided surgery trial, ${ }^{9}$ favorable emission profile $\left(\lambda_{\mathrm{ex}} 650 \mathrm{~nm} ; \lambda_{\mathrm{em}} 670 \mathrm{~nm}\right)$, high extinction coefficient $\left(\sim 250000 \mathrm{M}^{-1} \mathrm{~cm}^{-1}\right)$, high quantum yield in aqueous solutions $(\sim 0.25)$, and versatility as a synthetic platform. Nonetheless, the approach demonstrated here should also be easily translated to cyanine derivatives with a polymethine chain of different length. Variations in the chemical design of the Cy5-derivatives included the alkylation
Table 1. Characteristics of Evaluated Hybrid c[RGDyK] Tracers $^{a}$

\begin{tabular}{lcccc} 
dye & $\begin{array}{c}\text { net charge of } \\
\text { Cy5-derivative }\end{array}$ & $\begin{array}{c}\text { number of charges/ } \\
\text { Cy5-derivative }\end{array}$ & $\begin{array}{c}\text { logP Cy5- } \\
\text { derivative }\end{array}$ & $\begin{array}{c}\text { overall } \\
\text { charge } \\
\text { tracer }\end{array}$ \\
\hline 1 & na & na & -0.54 & -1 \\
2 & na & na & na & -1 \\
3 & na & na & na & +1 \\
4 & 0 & 2 & 0.52 & -1 \\
5 & +1 & 1 & 3.95 & 0 \\
6 & +2 & 2 & 0.27 & +1 \\
7 & -1 & 3 & -0.70 & -2 \\
8 & 0 & 2 & -0.14 & -1 \\
9 & +1 & 3 & -3.81 & 0 \\
10 & -2 & 4 & -1.93 & -3 \\
11 & -1 & 3 & -1.36 & -2 \\
12 & 0 & 4 & -4.71 & -1 \\
$a_{\text {na: }}$ & not applicable. & & & \\
\hline
\end{tabular}

of the indole-nitrogen and substitution of the aromatic ring. This systematic variation of the Cy5 design resulted in nine Cy5-derivatives with differences in overall charge $(-2$ to +2$)$, number of charges ( 1 to 4$)$, and predicted hydrophilicity $(\log \mathrm{P})$ $(-4.71$ to 3.95$){ }^{29}$

The different Cy5-derivatives were incorporated into a standardized hybrid imaging label comprising a peptide scaffold and a DTPA chelate. Subsequently, the hybrid labels were conjugated to $c[R G D y K]$, leading to the formation of the hybrid targeted tracers 4-12 (Figure 1). The conjugation of the hybrid label to the targeting peptide resulted in an overall charge for the complete tracer ranging from -3 to +1 , assuming a neutral charge for the $c[R G D y K]$ and a charge of -1 for DTPA (with or without chelated ${ }^{111} \mathrm{In}^{3+}$ ). ${ }^{30}$ The chemical characteristics of the tracers are included in Table 1 . All studied Cy5-derivatives incorporated into the hybrid tracer proved to be stable upon exposure to serum for $24 \mathrm{~h}$ (Figure S1). ${ }^{31}$ A hybrid label scaffold wherein no fluorescent dye was incorporated (1), a sole DTPA chelate (2), and a non- 
functionalized c[RGDyK $]$ peptide (3) were used as reference compounds.

The synthesized tracers and reference compounds were evaluated in vitro for their affinity for $\alpha_{v} \beta_{3}$-integrin. Flow cytometric analysis using epithelial-derived murine GE $\beta 3$ cells that stably overexpress $\alpha_{\mathrm{v}} \beta_{3}$-integrin revealed that conjugation of the hybrid label to $\mathrm{c}[\mathrm{RGDyK}]$ had little effect on the affinity for $\alpha_{\mathrm{v}} \beta_{3}$-integrin (Figure 2A). ${ }^{32}$ Most of the tracers possessed a

\begin{tabular}{cl}
\hline A & \\
\hline Tracer & Kd (nM) \\
\hline 1 & $44.2+/-5.9$ \\
2 & $138.1+/-27.2$ \\
3 & $38.8+/-4.3$ \\
4 & $50.2+/-20.7$ \\
5 & $36.7+/-15.1$ \\
6 & $35.6+/-12.4$ \\
7 & $21.9+/-5.9$ \\
8 & $29.1+/-15.1$ \\
9 & $33.1+/-11.3$ \\
10 & $84.2+/-16.7$ \\
11 & $85.2+/-23.5$ \\
12 & $142.3+/-42.0$ \\
\end{tabular}
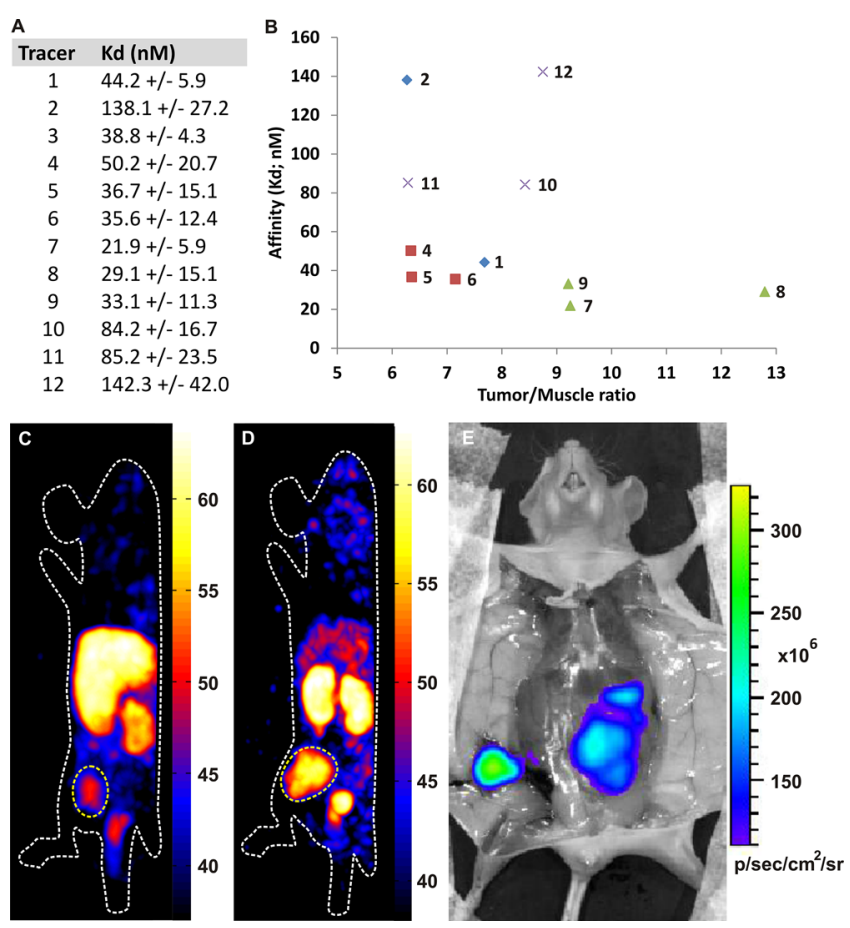

Figure 2. In vitro receptor affinity of the hybrid targeted tracers and in vivo SPECT imaging. (A) In vitro affinity (fluorescence) of the evaluated tracers toward $\alpha_{\mathrm{v}} \beta_{3}$-integrin determined by flow cytometric analysis (fluorescence). (B) Correlation between the in vitro affinity of the hybrid tracers for $\alpha_{\mathrm{v}} \beta_{3}$-integrin (fluorescence) and the $\mathrm{T} / \mathrm{M}$ ratio in vivo (radioisotope-based). Representative SPECT images of a 4T1tumor bearing mouse injected with a hybrid tracer showing (C) high liver uptake (5) and (D) low liver uptake (8). Contours of the mouse and tumor are represented by the dotted line. (E) T/M ratios of $>5$ (radioisotope-based) allowed accurate in vivo tumor visualization of the tumor via fluorescence imaging (representative image after injection of hybrid tracer 8 ).

similar affinity for $\alpha_{\mathrm{v}} \beta_{3}$-integrin as the unlabeled parental c[RGDyK] (3) $(38.6 \pm 4.3 \mathrm{nM})$, which could in turn be used to block the receptor interactions (Figure S2). Direct conjugation of the small DTPA-chelate to the lysine residue of the RGD-peptide (2) resulted in a drop in affinity to $138.1 \pm$ $27.2 \mathrm{nM}$. Introduction of the peptide-based scaffold of the hybrid label in 1 seemed to restore the initial affinity (44.2 \pm $5.9 \mathrm{nM})$. Strikingly, the presence of a single negatively charged sulfonate group on the aromatic indole moiety of the Cy5derivative (7-9) yielded a similar receptor affinity as obtained with the Cy5-derivatives that did not contain sulfonate groups on the indole moiety (4-6). Hybrid labels that included a Cy5derivative with two sulfonate groups (10-12) showed the lowest affinity (Figure 2A). An explanation for the lower affinity of the hybrid tracers that contain a Cy5-derivative wherein two sulfonate groups were included could not be found. Overall, 7 displayed the highest receptor affinity of the evaluated tracers $(21.9 \pm 5.9 \mathrm{nM})$.

In vivo evaluation of the hybrid tracers was performed using an orthotopic 4T1 breast cancer mouse model. ${ }^{10,33,34}$ Analysis of the biodistribution of $1 \mathrm{nmol}{ }^{111}$ In-labeled ( $1 \mathrm{MBq}$ ) hybrid tracer (4-12) or reference tracer $(1,2)$ was performed at $24 \mathrm{~h}$ post-injection to eliminate the presence of unwanted background activity caused by unbound circulating tracer. ${ }^{10}$ The biodistribution of the compounds could be visualized using whole-body SPECT imaging, as exemplified in representative images in Figure 2C and D (see Figure S3 for a complete overview of SPECT-based in vivo tracer distribution). The fluorescent signature also allowed clear tumor identification via fluorescence imaging, with low background fluorescence from the intestine (Figure 2E). Assessment of the percentage of the injected dose per gram of tissue $(\% \mathrm{ID} / \mathrm{g}$ ) showed substantial differences in the uptake of the tracers in the liver and kidneys as well as in the $\mathrm{T} / \mathrm{M}$ ratio (Figure 3 ).

To objectively assess the influence of the systematically varied dye structure on the in vivo behavior of the various hybrid tracers, an in vivo scoring system was developed. The biodistribution data of hybrid tracers 2 and 4-12 were scored relative to reference compound $\mathbf{1}$, which contained the hybrid scaffold without a fluorescent label (Table 2; for scoring parameters see SI). Significant improvements $(p \leq 0.05)$ in desired effects (such as increased tumor uptake or reduced nonspecific organ retention) are highlighted in green, whereas a significant undesired effect $(p \leq 0.05)$ (e.g., increase in background signal) is highlighted in red.

Compounds 4-11 showed superior tumor uptake compared to reference compound $\mathbf{1}$. All studied hybrid tracers provided a $\mathrm{T} / \mathrm{M}$-ratio $>5$ based on the detection of the radioisotope (Figure 3). In line with previous (clinical) studies, ${ }^{35}$ this indicates that there is sufficient contrast between the tumor and the surrounding tissue to allow for fluorescence-based imageguided surgery.

Table 2 and Figure 3 illustrate that incorporation of the group of Cy5-derivatives with the highest predicted LogP values (Table $1 ; 4-6$ ) resulted in a significant increase in nonspecific uptake in various tissues, which is line with previous findings using an alternative hydrophobic Cy5 dye with a similar $\log \mathrm{P}$ value. ${ }^{10}$ The addition of sulfonate groups to the aromatic moiety of the dyes lowered the predicted LogP (712; Table 1). In vivo this resulted in a reduction of the background signal while the tumor uptake was retained. Subsequently this yielded a higher imaging contrast (T/Mratio) (Table 2 and Figure 3). This effect was most prominent for the Cy5-derivatives with a single sulfonate group (7-9).

The renal accumulation of tracers 4-12 appeared to be related to the net charge of the Cy5-derivatives. In the series $5(+1)-8(0)-11(-1), 6(+2)-8(0)-10(-2)$, and $7(-1)$ $-8(0)-9(+1)$, the neutrally charged compound 8 displayed a lower kidney accumulation compared to either negatively or positively charged derivatives. While the neutrally charged 8 provides renal accumulation similar to that found for the nondye-containing 1 and 2, the neutral dyes 4 and 12 display a higher accumulation in the kidneys. In 4 , the $\mathrm{SO}_{3}{ }^{-}$moiety is on a "flexible" arm rather than one of the "rigid" aromatic units, a subtle difference that seems to induce renal accumulation. This effect is underlined by the fact that the series $4-7-\mathbf{1 0}$ displays the highest renal accumulation. In 12, the presence of multiple charged groups, even though the overall dye is neutrally charged, again seems to drive the renal accumulation 

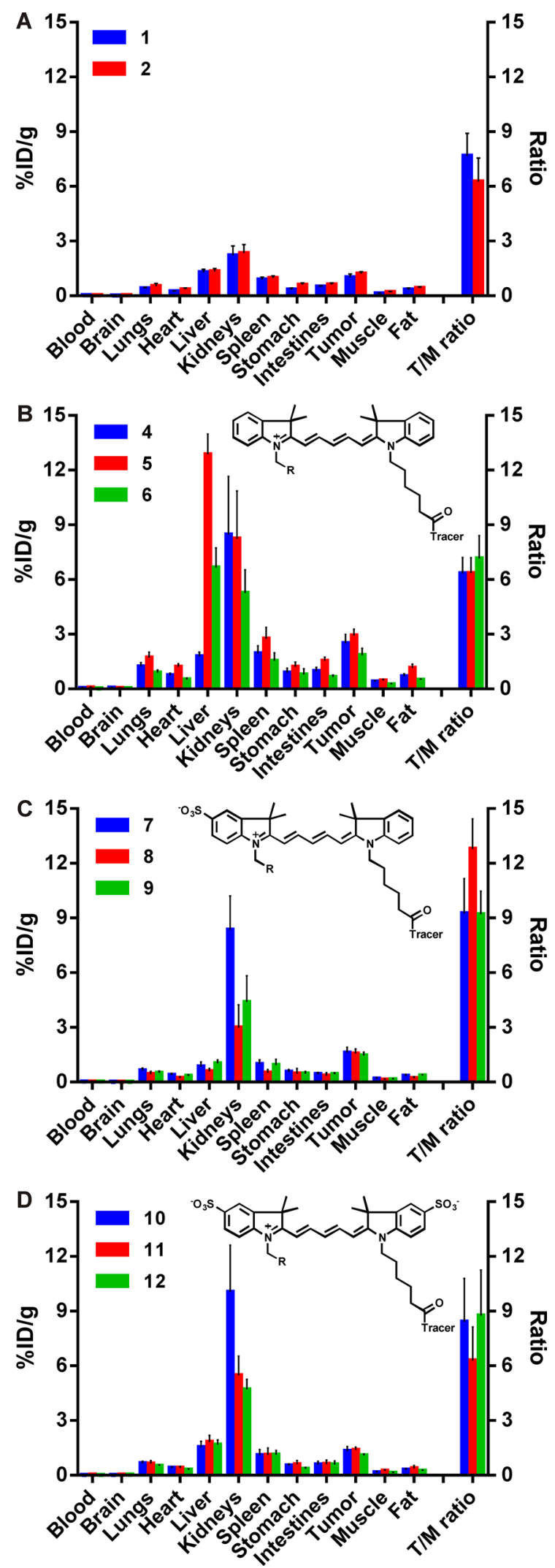

Figure 3. Radioisotope-based biodistribution of hybrid tracers 1, 2, and 4-12 in 4T1-tumor bearing mice at $24 \mathrm{~h}$ p.i. $(n=6$ for each tracer). (A) Reference compounds: hybrid label scaffold (1) and c[RGDyK]-DTPA (2). (B) Cy5-dyes without aromatic sulfonate moiety (4-6). (C) Cy5-dyes with one aromatic sulfonate moiety (79). (D) Cy5-dyes with two aromatic sulfonate moieties $(10-12) .(\mathrm{R}=$ see Figure 1A; $\mathrm{R}_{2}=-\left(\mathrm{CH}_{2}\right)_{3}-\mathrm{SO}_{3}{ }^{-}$, or $-\mathrm{H}$, or $\left.-\left(\mathrm{CH}_{2}\right)_{2} \mathrm{~N}^{+}\left(\mathrm{CH}_{3}\right)_{3}\right)$. compared to 8 . When combined, these observations show that accumulation of these compounds in the kidneys is influenced by the position as well as the number of charges present.

When combining the in vitro and in vivo analysis it can be concluded that the hybrid tracers with Cy 5 dyes that contain a single aromatic sulfonate moiety (7-9) showed the highest receptor affinity (Figure $2 \mathrm{~A}$ ), the most optimal tumor retention (Table 2), and the highest $\mathrm{T} / \mathrm{M}$ ratio (Figure $2 \mathrm{~B}$ and 3 ). Of these compounds, 8 stands out as it is the only hybrid tracer with overall positive in vivo characteristics relative to the reference compound (1) (Table 2). The Cy5 dyes without aromatic sulfonate moieties (4-6) maintained a high receptor affinity but yielded the lowest $\mathrm{T} / \mathrm{M}$ ratios as a result of increased nonspecific (Figure 3B). The Cy5 dyes with two aromatic sulfonate moieties (10-12) showed the lowest receptor affinity and T/M-ratios (Figures $2 \mathrm{~B}$ and $3 \mathrm{D}$ ).

In summary, it was shown that tracers with a fluorescent label that have a moderately negative hydrophilicity $(\log P)$, and balanced charge distribution, can be used to improve the biodistribution of the imaging tracer relative to the reference radiotracers (1 and 2). The uniquely designed matrix approach allowed the systematical evaluation of the different (hybrid) imaging tracers in vivo.

Scoring of the imaging tracers allowed us to isolate the effect of structural modification of the dyes on the in vivo characteristics of the hybrid tracer. In our view, this concept can have important implications for the design of alternative imaging tracers in the future. The modular design of the hybrid labels not only allows variation of the dye, but also allows variation of the chelate or targeting ligand. Hence the presented tracer optimization concept should also be applicable for the introduction of PET- or therapeutic isotopes as well as other targeting moieties directed against, e.g., cMET, CXCR4receptor, or PSMA. ${ }^{9,14,36}$ From this study it is evident that the net properties, and especially the overall charge and the charge distribution of the tracer, dictate the in vitro and in vivo behavior. This extrapolates into the statement: "There is no such thing as a universal imaging label that is suitable for all targeting moieties." In fact, it is highly likely that each individual receptor-targeted vector will require a systematic evaluation of the label that can realize specific imaging properties, being either fluorescent, radioactive, or hybrid.

\section{CONCLUSIONS}

Hybrid imaging labels were shown to provide combined quantitative assessment of both the affinity and in vivo distribution of the tracers. This enabled systematic assessment of the influence of the chemical modifications of the fluorescent dye. The developed matrix-based scoring system showed a large influence of relatively small changes in the chemical design of fluorescent labels. This approach not only resulted in an optimized $\alpha_{\mathrm{v}} \beta_{3}$-integrin targeted hybrid tracer (tracer 8), but also provides a systematic approach for the optimization of other (hybrid) fluorescent tracers.

\section{EXPERIMENTAL SECTION}

Detailed synthesis description of the hybrid targeted imaging agents and the in vitro and in vivo experiments is given in the Supporting Information. 
Table 2. Scoring of the Biodistribution of the Hybrid Tracers (Radioisotope-Based) Relative to Reference Compound $1^{a}$

$\begin{array}{lccccccccccccc} & \text { Blood } & \text { Brain } & \text { Lungs } & \text { Heart } & \text { Liver } & \text { Kidneys } & \text { Spleen } & \text { Stomach } & \text { Intestine } & \text { Tumor } & \text { Muscle } & \text { Fat } & \text { T/M } \\ \mathbf{1} & 1.00 & 1.00 & 1.00 & 1.00 & 1.00 & 1.00 & 1.00 & 1.00 & 1.00 & 1.00 & 1.00 & 1.00 & 1.00 \\ \mathbf{2} & 1.08 & 1.28 & 1.32 & 1.51 & 1.04 & 1.05 & 1.10 & 1.73 & 1.24 & 1.19 & 1.50 & 1.26 & 0.82 \\ \mathbf{4} & 2.18 & 2.64 & 3.05 & 3.18 & 1.38 & 3.81 & 2.19 & 2.62 & 1.97 & 2.45 & 2.97 & 2.01 & 0.83 \\ \mathbf{5} & 2.77 & 2.62 & 4.29 & 5.17 & 9.95 & 3.71 & 3.08 & 3.52 & 3.11 & 2.86 & 3.49 & 3.38 & 0.83 \\ \mathbf{6} & 1.17 & 1.47 & 2.22 & 2.21 & 5.14 & 2.37 & 1.74 & 2.29 & 1.33 & 1.81 & 1.94 & 1.50 & 0.93 \\ \mathbf{7} & 1.10 & 1.00 & 1.59 & 1.64 & 0.66 & 3.76 & 1.11 & 1.64 & 0.88 & 1.59 & 1.39 & 1.02 & 1.20 \\ \mathbf{8} & 1.22 & 0.72 & 1.10 & 0.96 & 0.46 & 1.35 & 0.58 & 1.36 & 0.73 & 1.53 & 0.95 & 0.65 & 1.66 \\ \mathbf{9} & 1.13 & 0.85 & 1.24 & 1.42 & 0.80 & 1.98 & 1.06 & 1.35 & 0.91 & 1.43 & 1.22 & 1.09 & 1.20 \\ \mathbf{1 0} & 0.76 & 0.97 & 1.63 & 1.75 & 1.20 & 4.52 & 1.22 & 1.52 & 1.18 & 1.30 & 1.23 & 0.91 & 1.10 \\ \mathbf{1 1} & 1.00 & 1.04 & 1.61 & 1.71 & 1.41 & 2.47 & 1.25 & 1.75 & 1.23 & 1.36 & 1.77 & 1.12 & 0.82 \\ \mathbf{1 2} & 0.70 & 1.08 & 1.29 & 1.31 & 1.30 & 2.12 & 1.26 & 1.06 & 1.19 & 1.06 & 0.99 & 0.69 & 1.14\end{array}$

${ }^{a}$ Significant increase in favorable uptake compared to $\mathbf{1}$ highlighted in green; significant increase in unfavorable uptake compared to $\mathbf{1}$ highlighted in red $(P<0.05) . \mathrm{T} / \mathrm{M}=$ tumor to muscle ratio.

\section{ASSOCIATED CONTENT}

\section{S Supporting Information}

The Supporting Information is available free of charge on the ACS Publications website at DOI: 10.1021/acs.bioconjchem.6b00093.

Materials and methods of the performed synthesis, characterization of the hybrid tracers, radiolabeling, in vitro evaluation and in vivo experiments (PDF)

\section{AUTHOR INFORMATION}

\section{Corresponding Author}

*E-mail: F.W.B.van_Leeuwen@lumc.nl.

Notes

The authors declare no competing financial interest.

\section{ACKNOWLEDGMENTS}

We thank M.N. van Oosterom for his assistance with the SPECT imaging. This research was financially supported by a Koningin Wilhelmina Fonds (KWF) translational research award (Grant No. PGF 2009-4344), a Netherlands Organization for Scientific Research STW-VIDI grant (Grant No. STW BGT11272), and a European Research Council under the European Union's Seventh Framework Program (FP7/20072013) grant (Grant No. 2012-306890) and the 2015-2016 Post-Doctoral Molecular Imaging Scholar Program Grant granted by the Society of Nuclear Medicine and Molecular imaging (SNMMI) and the Education and Research Foundation for Nuclear Medicine and Molecular Imaging.

\section{ABBREVIATIONS}

CXCR4, chemokine receptor 4; DOTA, 1,4,7,10-tetraazacyclododecane-1,4,7,10-tetraacetic acid; DTPA, diethylenetriamine penta-acetic acid; PET, positron emission tomography; PSMA, prostate specific membrane antigen; SPECT, single photon emission computed tomography; $\mathrm{T} / \mathrm{M}$ ratio, tumor-to-muscle ratio

\section{REFERENCES}

(1) van den Berg, N. S., Brouwer, O. R., Schaafsma, B. E., Matheron, H. M., Klop, W. M., Balm, A. J., van Tinteren, H., Nieweg, O. E., van Leeuwen, F. W. B., and Valdes Olmos, R. A. (2015) Multimodal
Surgical Guidance during Sentinel Node Biopsy for Melanoma: Combined Gamma Tracing and Fluorescence Imaging of the Sentinel Node through Use of the Hybrid Tracer Indocyanine Green-TcNanocolloid. Radiology 275, 521-529.

(2) KleinJan, G. H., van den Berg, N. S., Brouwer, O. R., de Jong, J., Acar, C., Wit, E. M., Vegt, E., van der Noort, V., Valdes Olmos, R. A., van Leeuwen, F. W. B., et al. (2014) Optimisation of Fluorescence Guidance During Robot-assisted Laparoscopic Sentinel Node Biopsy for Prostate Cancer. Eur. Urol. 66, 991-998.

(3) Brouwer, O. R, van den Berg, N. S., Matheron, H. M., van der Poel, H. G., van Rhijn, B. W., Bex, A., van Tinteren, H., Valdes Olmos, R. A., van Leeuwen, F. W. B., and Horenblas, S. (2014) A hybrid radioactive and fluorescent tracer for sentinel node biopsy in penile carcinoma as a potential replacement for blue dye. Eur. Urol. 65, 6009.

(4) van Leeuwen, F. W., Hardwick, J. C., and van Erkel, A. R. (2015) Luminescence-based Imaging Approaches in the Field of Interventional Molecular Imaging. Radiology 276, 12-29.

(5) Kuil, J., Velders, A. H., and van Leeuwen, F. W. B. (2010) Multimodal tumor-targeting peptides functionalized with both a radioand a fluorescent label. Bioconjugate Chem. 21, 1709-19.

(6) Schottelius, M., and Wester, H. J. (2009) Molecular imaging targeting peptide receptors. Methods 48, 161-177.

(7) Choi, H. S., Gibbs, S. L., Lee, J. H., Kim, S. H., Ashitate, Y., Liu, F., Hyun, H., Park, G., Xie, Y., Bae, S., et al. (2013) Targeted zwitterionic near-infrared fluorophores for improved optical imaging. Nat. Biotechnol. 31, 148-53.

(8) Conner, K. P., Rock, B. M., Kwon, G. K., Balthasar, J. P., Abuqayyas, L., Wienkers, L. C., and Rock, D. A. (2014) Evaluation of near infrared fluorescent labeling of monoclonal antibodies as a tool for tissue distribution. Drug Metab. Dispos. 42, 1906-13.

(9) Burggraaf, J., Kamerling, I. M. C., Gordon, P. B., Schrier, L., de Kam, M. L., Kales, A. J., Bendiksen, R., Yazdanfar, S., Langers, A. M. J., Torheim, G., et al. (2015) Detection of colorectal neoplasia in vivo in humans using an intravenously administered fluorescent peptide targeted against c-Met and fluorescence colonoscopy; a proof of concept study. Nat. Med. 21, 955-961.

(10) Bunschoten, A., Buckle, T., Visser, N., Kuil, J., Yuan, H., Josephson, L., Vahrmeijer, A. L., and van Leeuwen, F. W. B. (2012) Multimodal interventional molecular imaging of tumor margins and distant metastases by targeting the $\alpha \mathrm{v} \beta 3$ integrin. ChemBioChem 13, 1039-1045.

(11) Weissleder, R. (2001) A clearer vision for in vivo imaging. Nat. Biotechnol. 19, 316-7.

(12) Escobedo, J. O., Rusin, O., Lim, S., and Strongin, R. M. (2010) NIR dyes for bioimaging applications. Curr. Opin. Chem. Biol. 14, 6470. 
(13) Kuil, J., Buckle, T., Yuan, H., Oishi, S., Fujii, N., Josephson, L., van Leeuwen, F. W. B., and van den Berg, N. S. (2011) Synthesis and in vitro evaluation of a bimodal CXCR4 antagonistic peptide. Bioconjugate Chem. 22, 859-864.

(14) Kuil, J., Buckle, T., and van Leeuwen, F. W. B. (2012) Imaging agents for the chemokine receptor 4 (CXCR4). Chem. Soc. Rev. 41, 5239-5261.

(15) Yin, L., Wang, W., Wang, S., Zhang, F., Zhang, S., and Tao, N. (2015) How does fluorescent labeling affect the binding kinetics of proteins with intact cells? Biosens. Bioelectron. 66, 412-6.

(16) Weis, S. M., and Cheresh, D. A. (2011) alphaV integrins in angiogenesis and cancer. Cold Spring Harbor Perspect. Med. 1, a006478.

(17) Brooks, P. C., Clark, R. A., and Cheresh, D. A. (1994) Requirement of vascular integrin alpha $\mathrm{v}$ beta 3 for angiogenesis. Science 264, 569-71.

(18) Huveneers, S., Truong, H., and Danen, H. J. (2007) Integrins: signaling, disease, and therapy. Int. J. Radiat. Biol. 83, 743-51.

(19) Desgrosellier, J. S., and Cheresh, D. A. (2010) Integrins in cancer: biological implications and therapeutic opportunities. Nat. Rev. Cancer 10, 9-22.

(20) Ye, Y., and Chen, X. (2011) Integrin targeting for tumor optical imaging. Theranostics 1, 102-26.

(21) Gaertner, F. C., Kessler, H., Wester, H. J., Schwaiger, M., and Beer, A. J. (2012) Radiolabelled RGD peptides for imaging and therapy. Eur. J. Nucl. Med. Mol. Imaging 39 (Suppl 1), S126-38.

(22) Haubner, R., Weber, W. A., Beer, A. J., Vabuliene, E., Reim, D., Sarbia, M., Becker, K. F., Goebel, M., Hein, R., Wester, H. J., et al. (2005) Noninvasive visualization of the activated alphavbeta3 integrin in cancer patients by positron emission tomography and [18F]Galacto-RGD. PLoS Med. 2, e70.

(23) Dearling, J. L., Barnes, J. W., Panigrahy, D., Zimmerman, R. E., Fahey, F., Treves, S. T., Morrison, M. S., Kieran, M. W., and Packard, A. B. (2013) Specific uptake of 99mTc-NC100692, an alphavbeta3targeted imaging probe, in subcutaneous and orthotopic tumors. Nucl. Med. Biol. 40, 788-94.

(24) Mena, E., Owenius, R., Turkbey, B., Sherry, R., Bratslavsky, G., Macholl, S., Miller, M. P., Somer, E. J., Lindenberg, L., Adler, S., et al. (2014) [(1)(8)F]fluciclatide in the in vivo evaluation of human melanoma and renal tumors expressing alphavbeta 3 and alpha vbeta 5 integrins. Eur. J. Nucl. Med. Mol. Imaging 41, 1879-88.

(25) Wan, W., Guo, N., Pan, D., Yu, C., Weng, Y., Luo, S., Ding, H., Xu, Y., Wang, L., Lang, L., et al. (2013) First experience of 18F-alfatide in lung cancer patients using a new lyophilized kit for rapid radiofluorination. J. Nucl. Med. 54, 691-8.

(26) Haubner, R., Wester, H. J., Reuning, U., SenekowitschSchmidtke, R., Diefenbach, B., Kessler, H., Stocklin, G., and Schwaiger, M. (1999) Radiolabeled alpha(v)beta3 integrin antagonists: a new class of tracers for tumor targeting. J. Nucl. Med. 40, 1061-71.

(27) van Hagen, P. M., Breeman, W. A., Bernard, H. F., Schaar, M., Mooij, C. M., Srinivasan, A., Schmidt, M. A., Krenning, E. P., and de Jong, M. (2000) Evaluation of a radiolabelled cyclic DTPA-RGD analogue for tumour imaging and radionuclide therapy. Int. J. Cancer 90, 186-98.

(28) Lohof, E., Planker, E., Mang, C., Burkhart, F., Dechantsreiter, M. A., Haubner, R., Wester, H. J., Schwaiger, M., Holzemann, G., Goodman, S. L., et al. (2000) Carbohydrate Derivatives for Use in Drug Design: Cyclic alpha(v)-Selective RGD Peptides. Angew. Chem., Int. Ed. 39, 2761-2764.

(29) http://www.molinspiration.com/cgi-bin/properties; Accessed 11-09-2015.

(30) Maecke, H. R., Riesen, A., and Ritter, W. (1989) The molecular structure of indium-DTPA. J. Nucl. Med. 30, 1235-9.

(31) Hyun, H., Wada, H., Bao, K., Gravier, J., Yadav, Y., Laramie, M., Henary, M., Frangioni, J. V., and Choi, H. S. (2014) Phosphonated near-infrared fluorophores for biomedical imaging of bone. Angew. Chem., Int. Ed. 53, 10668-72.

(32) Huveneers, S., van den Bout, I., Sonneveld, P., Sancho, A., Sonnenberg, A., and Danen, E. H. (2007) Integrin alpha v beta 3 controls activity and oncogenic potential of primed c-Src. Cancer Res. 67, 2693-700.

(33) Pulaski, B. A., and Ostrand-Rosenberg, S. (2001) Mouse 4T1 breast tumor model. In Current Protocols in Immunology, Chapter 20, Unit 20 2, John Wiley \& Sons, Inc., Hoboken, NJ, DOI: 10.1002/ 0471142735.im2002s39

(34) Sloan, E. K., Pouliot, N., Stanley, K. L., Chia, J., Moseley, J. M., Hards, D. K., and Anderson, R. L. (2006) Tumor-specific expression of alphavbeta3 integrin promotes spontaneous metastasis of breast cancer to bone. Breast Cancer Res. 8, R20.

(35) Schaafsma, B. E., Verbeek, F. P., Rietbergen, D. D., van der Hiel, B., van der Vorst, J. R., Liefers, G. J., Frangioni, J. V., van de Velde, C. J., van Leeuwen, F. W. B., and Vahrmeijer, A. L. (2013) Clinical trial of combined radio- and fluorescence-guided sentinel lymph node biopsy in breast cancer. Br. J. Surg. 100, 1037-44.

(36) Osborne, J. R., Akhtar, N. H., Vallabhajosula, S., Anand, A., Deh, K., and Tagawa, S. T. (2013) Prostate-specific membrane antigenbased imaging. Urol. Oncol. 31, 144-54. 